\section{Warming goal: still the best indicator}

David Victor and Charles Kennel challenge the practice of using global mean temperature as the main measure of danger from climate change (Nature 514, $30-31 ; 2014)$. On the basis of 40 years of science and policy research, there are good reasons why this temperature is the favoured indicator.

It can be related through climate models to the regional impacts and risks that drive public concern (see go.nature. $\mathrm{com} / 5 \mathrm{chktj}$ ). It is indeed "related only probabilistically to emissions", but the authors' best indicator - carbon dioxide concentration - is related only probabilistically to impacts and risks, except in the case of ocean acidification. As for ocean heat content, its trend experiences interruptions much like the global mean temperature, and bears no direct relationship to most impacts and risks.

Compared with other proposals, global mean temperature is more closely related to outcomes for people and ecosystems. Without such a goal, we shall never know how much reduction in emissions is sufficient.

Michael Oppenheimer Princeton University, New Jersey, USA. omichael@princeton.edu

\section{Warming goal: clear link to emissions}

David Victor and Charles Kennel argue that aiming to keep average global warming within $2^{\circ} \mathrm{C}$ of pre-industrial temperatures is neither politically nor scientifically useful (Nature 514, 30-31; 2014). I disagree: global temperature change is the closest thing we have to a metric with a clear link to emissions; it can also be related quantitatively to a range of local climate impacts.

Because global temperature seems to respond linearly to cumulative emissions of carbon dioxide (H. D. Matthews et al. Nature 459, 829-832; 2009), policies to cut emissions should also reduce global temperature change. This offers a simple framework for estimating a global carbon budget that contains warming to within $2^{\circ} \mathrm{C}$.

Policy goals should not have adverse effects on human and environmental welfare. Using global temperature avoids these too, because it seems to be an indicator of the extent of local climate changes (see, for example, M. Markovic et al. Clim. Change 120, 197-210; 2013). Furthermore, the average global temperature over decades relates well to many climate impacts and to Victor and Kennel's 'vital signs' of planetary health (National Research Council Climate Stabilization Targets National Academies Press, 2011).

Now that the international community has finally coalesced around the $2{ }^{\circ} \mathrm{C}$ goal, compelling reasons are needed to interrupt this momentum.

H. Damon Matthews Concordia University, Montreal, Canada. damon.matthews@concordia.ca

\section{Open access to Earth land-cover map}

China last month donated to the United Nations the first open-access, high-resolution map of Earth's land cover, as a contribution towards global sustainable development and combating climate change.

The map, known as GlobeLand30, comprises data sets collected at 30-metre resolution - more than ten times that of previous data sets. These data sets will be valuable for monitoring environmental changes and for resource management at global, regional and local scales (see also M. A. Wulder and N. C. Coops Nature 513, 30-31; 2014).

The GlobeLand 30 data sets are freely available and comprise ten types of land cover, including forests, artificial surfaces and wetlands, for the years 2000 and
2010. They were extracted from more than 20,000 Landsat and Chinese $\mathrm{HJ}$-1 satellite images (see www.globallandcover.com).

GlobeLand30 will promote scientific data sharing in the fields of Earth observation and geospatial sciences.

Chen Jun National Geomatics Center of China, Beijing, China. chenjun@nsdi.gov.cn Yifang Ban KTH Royal Institute of Technology, Stockholm, Sweden. Songnian Li Ryerson University, Toronto, Ontario, Canada.

\section{Sustainability: root targets in consensus}

Mark Stafford-Smith

urges scientists to engage more effectively with the

United Nations' Sustainable Development Goals to ensure that their environmental targets are quantifiable (Nature 513, 281; 2014). When stakeholder values are diverse and passionately defended, however, such targets may not be easily agreed leading to stalled negotiations and stagnant progress on issues of global significance.

In our view, building consensus over desirable environmental outcomes would be a better approach. This involves analysing different possible outcomes, understanding decision-making processes and improving communication among stakeholders who have conflicting interests.

Initiatives such as Future Earth and the Intergovernmental Platform on Biodiversity and Ecosystem Services are helping scientists to engage with international environmental policy. In the ongoing negotiations over the Sustainable Development Goals, scientists need to move on from simple information provision and help to develop appropriate policies. Sean Maxwell* University of Queensland, Brisbane, Australia. smaxwell@uq.edu.au ${ }^{*}$ On behalf of 10 correspondents (see go.nature.com/tqxjyj for full list).

\section{Stop the cuts, not the evaluations}

Amaya Moro-Martin asserts that the European Science Foundation (ESF) supported a "flawed

evaluation process" for research in Portugal (Nature 514, 141; 2014).

This unsubstantiated allegation undermines the foundation's work and is detrimental to the many excellent reviewers and panel members involved in the evaluation process.

The ESF champions the benefits to society from investments in research. We are very concerned about the increased pressure on many national science budgets. However, we believe that peer review, despite its limitations, is the most meritocratic and evidence-based approach to resource allocation. The work of those public-spirited scientists willing to give their time and energy to the peer-review process must be acknowledged, respected and supported. They should be allowed to undertake their work without interference.

During the course of the independent research evaluation implemented for the Foundation for Science and Technology in Portugal, the ESF has witnessed an unprecedented level of direct interference with peers and panel members in the performance of their work. Even while the review process is ongoing, many have received intimidating communications designed to discourage them from completing their agreed tasks. This practice is unacceptable and damaging to science.

It is in this context that we respond to Moro-Martin's remark. Although no evaluation process is perfect, it is the most independent system yet devised. The ESF has carried out this evaluation project in accordance with good practice (see go.nature. com/o4xfuz; to be updated on completion of the project). Jean-Claude Worms, Jane Swift ESF, Strasbourg, France. jswift@esf.org 